Vietnam Journal of Mechanics, VAST, Vol.41, No. 4 (2019), pp. 301 -317

DOI: https://doi.org/10.15625/0866-7136/13356

\title{
DIFFERENTIAL EQUATIONS OF MOTION IN MATRIX FORM OF MULTIBODY SYSTEMS DRIVEN BY ELECTRIC MOTORS
}

\author{
Nguyen Quang Hoang ${ }^{1, *}$, Vu Duc Vuong ${ }^{2}$ \\ ${ }^{1}$ Hanoi University of Science and Technology, Vietnam \\ ${ }^{2}$ Thai Nguyen University of Technology, Vietnam \\ *E-mail: hoang.nguyenquang@hust.edu.vn
}

Received: 25 April 2019 / Published online: 20 October 2019

\begin{abstract}
This paper presents the dynamic model of multibody systems driven by electric motors, the so-called electromechanical systems. The mechanical systems considered in this study include an open loop and/or a closed loop, a full-actuated and an underactuated one. The dynamic model of this electromechanical systems is established in matrix form by applying the Lagrangian equation with and without multipliers and substructure method. With this approach it is easy to obtain the differential equation of motion of the electro-mechanical systems based on the corresponding differential equations of the purely available mechanical system. These obtained equations describe the electromechanical systems in engineering better in case the systems are purely described by mechanical equations. The differential equations of serial and parallel manipulators, slider-crank mechanism, and overhead crane driven by electric motors are established as illustrated examples. In addition, a simplified dynamic model obtained by neglecting of current variation is also validated by numerical simulations.
\end{abstract}

Keywords: multibody system, electromechanical system, equations of motion, dynamic models, underactuated system.

\section{INTRODUCTION}

Most of the industrial electromechanical systems may be considered as mechanical multibody systems driven by electric motors - also called actuators. The inputs of these systems are normally voltage or currents applied to motors and its output are the motion of the mechanical multibody systems (MBS). Some of examples of electromechanical systems can be listed as overhead cranes, serial or parallel robotic manipulators. Dynamic models of this system are an important base for analysis, simulation, controller design as well as numerical testing with different excitations, testing control laws, and optimization. 
The mechanical part of an industrial motion system is normally described by an open-loop or a closed-loop MBS, which is a set of bodies interconnected by joints. Modelling and simulation of an MBS has attracted numerous researchers. Many works deal with modelling of MBS [1-20]. There are some approaches proposed in literature to formulate dynamic equations of an MBS including Newton-Euler equations, Lagrangian formulation, principle of virtual work, Jourdain principle, and Kane equation [1-17]. In most of the mentioned works, gear transmission and dynamics of actuators are neglected. Thus, the equations describing industrial electromechanical systems using only the MBS model are not really close to real systems.

There are only a few studies showing the construction of dynamic models for the electromechanical system [21-28]. In [21,22], two methods of linear graph and bond graph theories were presented for modelling of electromechanical systems. Even though bond graphs could be obtained for the system, their complexity and their nature prevent us from easily generating the equations. Bond graphs are interesting for showing energy interactions between the different parts of the model. However, they suffer from obvious limitations as soon as complex 3D mechanical systems are considered. In [24], authors combined a linear graph theory with symbolic programming to model the flexible multibody mechatronic systems. In the study [23], authors extended the recursive dynamic formulation to model a mechanical system containing an electric DC motor. In [25], the driving torque is assigned to be proportional to the voltage applied to the motor, where the damping due to the armature resistance of the motor is ignored. In the work [26], methodologies for the development of formalisms and software for modelling and simulation of multibody and mechatronic systems was presented and illustrated by examples from the field of automobile and robotics.

The present paper highlights the dynamic modelling of MBS with a closed loop and/or an open loop driven by electric DC motors, one of the most popular electrical motors. The whole system is divided into three parts: an MBS, massless gear transmissions, and electric motors. For the multiboby system the generalized coordinates and the Lagrangian formulation are used to establish the equations of motion. In the cases of closed loop MBS, the differential equations are combined with the constraint equations, the dynamic model of a system is presented in differential algebraic equations form (DAEs). The equation describing the massless gear transmission is obtained by the power balance at input and output of the gearbox. And the dynamic equations of electric motors are derived, mainly based on the angular momentum law combined with the Kirchoffs laws. The obtained equations are then simplified with the assumption that the electrical time constant is much smaller than the mechanical time constant.

The main contributions of this paper are to provide the matrix form of equations of motion for a closed loop MBS driven by electric motors by introducing a matrix $\mathbf{Z}$, that describes the relation between redundant and active coordinates. In this way, it is easy to obtain the equation of motion for electromechanical systems from pure mechanical ones. Equations describing subsystems are written in matrix form. Therefore, they are easy to transform by matrix multiplication and addition, and it is also easy to simulate on the computer by software tools such as Maple. In addition, the paper also validated a simplified dynamic model by using numerical simulations. 
The remaining of this paper is organized as follows: Section 2 presents a dynamic modelling of mechanical systems actuated by electric motors. Section 3 presents some examples of modelling of systems in industrial application such as overhead cranes, robotic manipulators, a slider-crank mechanism and a 3RRR planar parallel manipulator. Section 4 shows the validation of simplified model by numerical simulations. Finally, the conclusion is given in Section 5.

\section{DYNAMIC MODEL OF MECHANICAL SYSTEMS DRIVEN BY ELECTRIC MOTORS}

This section presents a method for establishing motion equations of electromechanical systems in engineering by substructure method and Lagrangian formulation. The whole system is split into mechanical sub-systems and electric motors which are coupled by massless gear-box transmission. Firstly, the equations for each subsystem are given. Then they are combined by using matrix multiplication and addition to obtain the final equations that describe the relationship between input voltages and the motion of the systems.

\subsection{Dynamic model of mechanical systems}

Structurally, mechanical systems can be classified into a tree/serial and a closed-loop type. Closed-loop systems can be split into two or more tree structures by joint cutting. When the controlling issue is considered, a mechanical system can be classified into fullactuated systems if the number of motors is equal to the number of degrees of freedom and under-actuated one if the number of motors is less than the number of degrees of freedom. There are many methods for establishing equations of motion of mechanical systems presented in some references $[1,2,4,5]$.

Lets consider an open-loop mechanical system having $n$ DOFs with holonomic constraints. Let $\mathbf{q}=\left[q_{1}, q_{2}, \ldots, q_{m}\right]^{T}, m=n$, be generalized coordinates of the system. By using Lagrangian formulation, the equations of motion of this system are given by

$$
\frac{\mathrm{d}}{\mathrm{d} t}\left(\frac{\partial T}{\partial \dot{\mathbf{q}}}\right)^{T}-\left(\frac{\partial T}{\partial \mathbf{q}}\right)^{T}=\mathbf{Q}-\left(\frac{\partial \Pi}{\partial \mathbf{q}}\right)^{T} .
$$

Eq. (1) are rewritten in matrix form as

$$
\mathbf{M}(\mathbf{q}) \ddot{\mathbf{q}}+\mathbf{C}(\mathbf{q}, \dot{\mathbf{q}}) \dot{\mathbf{q}}+\mathbf{D} \dot{\mathbf{q}}+\mathbf{g}(\mathbf{q})=\mathbf{B} \tau_{2} \text {. }
$$

In above equations, $T=\frac{1}{2} \dot{\mathbf{q}}^{T} \mathbf{M}(\mathbf{q}) \dot{\mathbf{q}}$ is the kinetic energy; $\mathbf{M}(\mathbf{q})$ the $m \times m$ mass matrix that is hessian matrix of kinetic energy $T$ with respect to $\dot{\mathbf{q}} ; \mathbf{M}(\mathbf{q})=\partial[T / \partial \dot{\mathbf{q}}]^{T} / \partial \dot{\mathbf{q}} ; \Pi=$ $\Pi(\mathbf{q})$ the potential energy; $\mathbf{g}(\mathbf{q})=[\partial \Pi / \partial \mathbf{q}]^{T} ; \mathbf{D}$ is the damping matrix; $\mathbf{Q}$ is the vector of generalized forces of non-conservative forces.

Similarly, for a closed loop mechanical system having $n$ DOFs with holonomic constraints, let $\mathbf{q}=\left[q_{1}, q_{2}, \ldots, q_{m}\right]^{T}, m>n$ be redundant generalized coordinates of the system. By using Lagrangian formulation with multipliers, the equation of motion of this system and the constrained equations are given by 


$$
\begin{aligned}
& \frac{\mathrm{d}}{\mathrm{d} t}\left(\frac{\partial T}{\partial \dot{\mathbf{q}}}\right)^{T}-\left(\frac{\partial T}{\partial \mathbf{q}}\right)^{T}=\mathbf{Q}-\boldsymbol{\Phi}_{q}^{T}(\mathbf{q}) \boldsymbol{\lambda}-\left(\frac{\partial \Pi}{\partial \mathbf{q}}\right)^{T}, \\
& \boldsymbol{\phi}(\mathbf{q})=\mathbf{0} .
\end{aligned}
$$

Eq. (3) is rewritten in matrix form as:

$$
\begin{aligned}
& \mathbf{M}(\mathbf{q}) \ddot{\mathbf{q}}+\mathbf{C}(\mathbf{q}, \dot{\mathbf{q}}) \dot{\mathbf{q}}+\mathbf{D} \dot{\mathbf{q}}+\mathbf{g}(\mathbf{q})+\mathbf{\Phi}^{T}(\mathbf{q}) \boldsymbol{\lambda}=\mathbf{B} \tau_{2}, \\
& \boldsymbol{\phi}(\mathbf{q})=\mathbf{0},
\end{aligned}
$$

where $\mathbf{Q}=\mathbf{B} \tau_{2}, \mathbf{B}$ is the matrix related to the control input arrangement and $\tau_{2}$ the vector force/torque in the actuated joints. $\tau_{2}=\left[\tau_{2,1}, \tau_{2,2}, \ldots, \tau_{2, n}\right]^{T}$ - torque/force at the output of transmission. $\lambda=\left[\begin{array}{llll}\lambda_{1} & \lambda_{2} \ldots \lambda_{r}\end{array}\right]^{T}$ is the vector of Lagrangian multipliers, $r=m-n$; $\boldsymbol{\phi}(\mathbf{q})=\mathbf{0}$, with $\boldsymbol{\phi}=\left[\begin{array}{llll}\phi_{1} & \phi_{2} \ldots \phi_{r}\end{array}\right]^{T}$ including $m-n$ constraint equations; $\boldsymbol{\Phi}(\mathbf{q})=\partial \boldsymbol{\phi} / \partial \mathbf{q}$ is the $r \times m$ Jacobian matrix.

Matrix $\mathbf{C}(\mathbf{q}, \dot{\mathbf{q}})$ can be determined from the mass matrix $\mathbf{M}(\mathbf{q})$ using the Kronecker product [17] or the Christoffel formula [5] as follows

$$
\mathbf{C}(\mathbf{q}, \dot{\mathbf{q}})=\left\{c_{i j}(\mathbf{q}, \dot{\mathbf{q}})\right\}, \quad c_{i j}(\mathbf{q}, \dot{\mathbf{q}})=\frac{1}{2} \sum_{k=1}^{m}\left(\frac{\partial m_{i j}}{\partial q_{k}}+\frac{\partial m_{i k}}{\partial q_{j}}-\frac{\partial m_{j k}}{\partial q_{i}}\right) \dot{q}_{k} .
$$

Noting that the mass matrix $\mathbf{M}(\mathbf{q})$ is a symmetric one, and the matrix $\mathbf{C}(\mathbf{q}, \dot{\mathbf{q}})$ determined by (6) guarantees the skew-symmetric property of the matrix $\dot{\mathbf{M}}(\mathbf{q})-2 \mathbf{C}(\mathbf{q}, \dot{\mathbf{q}})$ [5]. This property plays an important role in control design for the system.

So, with the Lagrangian equation the differential equation of motion of the system is obtained if we know the kinetic energy, the potential energy of the system, and the generalized forces. In order to distinguish fully- and under- actuated system, the generalized coordinates are split into groups $\mathbf{q}=\left[q_{1}, q_{2}, \ldots, q_{m}\right]^{T}=\left[\mathbf{q}_{a}^{T}, \mathbf{q}_{p}^{T}\right]^{T}$, in which $\mathbf{q}_{a}$ is active coordinates and $\mathbf{q}_{p}$ is passive coordinates. In all cases of open and closed loop systems, they are fully-actuated if $\operatorname{dim} \mathbf{q}_{a}=\operatorname{dim} \tau_{2}=n$ and underactuated if $\operatorname{dim} \mathbf{q}_{a}=\operatorname{dim} \tau_{2}<n$.

\subsection{Dynamic model of electric motors and gear transmission}

Neglecting the mass and power loss, the constraint equations of the gear transmission of $n$ motors are written as follows

$$
\mathbf{R}_{G} \dot{\mathbf{q}}_{a} \equiv \mathbf{R}_{G} \dot{\boldsymbol{\theta}}=\dot{\boldsymbol{\theta}}_{m}, \quad \tau_{2}=\mathbf{R}_{G} \boldsymbol{\tau}_{1} .
$$

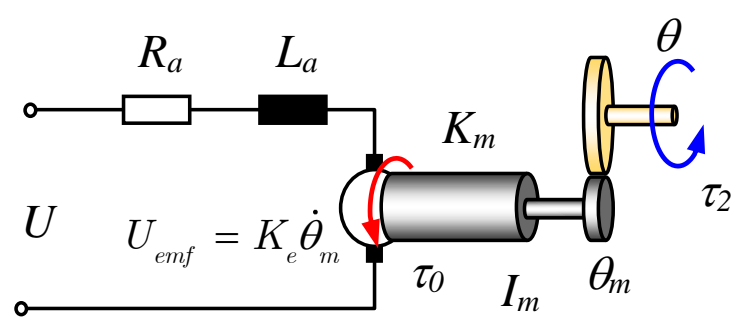

Fig. 1. Diagram of a motor and gear reducer 
Dynamics of actuators (electric motors - Fig. 1) is described by the mechanical and electrical equations $[15,26]$. By applying the angular momentum theorem for rotor and Kirchhoff voltage law of $n$ motors, ones obtain

$$
\begin{aligned}
\mathbf{I}_{m} \ddot{\boldsymbol{\theta}}_{m}+\mathbf{D}_{m} \dot{\boldsymbol{\theta}}_{m} & =\boldsymbol{\tau}_{0}-\boldsymbol{\tau}_{1}, \\
\mathbf{L}_{a} \frac{\mathrm{d}}{\mathrm{d} t} \mathbf{i}+\mathbf{R}_{a} \mathbf{i} & =\mathbf{u}-\mathbf{u}_{e} .
\end{aligned}
$$

The mechanical and electrical interaction of $n$ motors is shown by the relationship between current and motor torque $[15,26]$; between motor speed and EMFs voltages as follows

$$
\boldsymbol{\tau}_{0}=\mathbf{K}_{m} \mathbf{i}, \quad \mathbf{u}_{e}=\mathbf{K}_{e} \dot{\boldsymbol{\theta}}_{m} .
$$

In the above equations the following notations are used: $\tau_{1}=\left[\tau_{1,1}, \tau_{1,2}, \ldots, \tau_{1, n}\right]^{T}$ - Torque/force at the input of transmission; $\tau_{0}=\left[\tau_{0,1}, \tau_{0,2}, \ldots, \tau_{0, n}\right]^{T}$ - Torque/force of the DC motor; $\mathbf{R}_{G}=\operatorname{diag}\left(r_{1}, r_{2}, \ldots, r_{n}\right), r_{i}=\theta_{i} / \theta_{m, i}$ - Matrix of gear reduction ratio; $\mathbf{I}_{m}=\operatorname{diag}\left(I_{m, 1}, I_{m, 2}, \ldots, I_{m, n}\right)$ - Moment of inertia of rotors; $\mathbf{L}_{a}=\operatorname{diag}\left(L_{a, 1}, L_{a, 2}, \ldots, L_{a, n}\right)$ - Motor coil inductances; $\mathbf{R}_{a}=\operatorname{diag}\left(R_{a, 1}, R_{a, 2}, \ldots, R_{a, n}\right)$ - Motor coil resistances; $\mathbf{K}_{e}=$ $\operatorname{diag}\left(K_{e, 1}, K_{e, 2}, \ldots, K_{e, n}\right)$ - Back-emf constants; $\mathbf{K}_{m}=\operatorname{diag}\left(K_{m, 1}, K_{m, 2}, \ldots, K_{m, n}\right)$ - Motor torque constants; $\mathbf{u}=\left[U_{1}, U_{2}, \ldots, U_{n}\right]^{T}$ - Motor input voltages; $\mathbf{i}=\left[i_{1}, i_{2}, \ldots, i_{n}\right]^{T}$ - Currents in electric motors; $\mathbf{D}_{m}=\operatorname{diag}\left(b_{1}, b_{2}, \ldots, b_{n}\right)$ - Viscous coefficients of motor shafts.

\subsection{Dynamic model of electromechanical systems}

In order to get the dynamic model in compact form the constraint forces/moment $\tau_{1}, \tau_{2}$ will be eliminated from Eqs. (2), (8). By substituting (7) and (10) into (8) one gets

$$
\begin{gathered}
\mathbf{I}_{m} \ddot{\boldsymbol{\theta}}_{m}+\mathbf{D}_{m} \dot{\boldsymbol{\theta}}_{m}=\mathbf{K}_{m} \mathbf{i}-\boldsymbol{\tau}_{1}, \\
\mathbf{L}_{a} \frac{\mathrm{d}}{\mathrm{d} t} \mathbf{i}+\mathbf{R}_{a} \mathbf{i}=\mathbf{u}-\mathbf{K}_{e} \dot{\boldsymbol{\theta}}_{m} .
\end{gathered}
$$

Multiplying from left (11) with matrix $\mathbf{R}_{G}$ yields

$$
\mathbf{R}_{G} \mathbf{I}_{m} \ddot{\boldsymbol{\theta}}_{m}+\mathbf{R}_{G} \mathbf{D}_{m} \dot{\boldsymbol{\theta}}_{m}=\mathbf{R}_{G} \mathbf{K}_{m} \mathbf{i}-\mathbf{R}_{G} \boldsymbol{\tau}_{1} .
$$

By substituting $\dot{\boldsymbol{\theta}}_{m}=\mathbf{R}_{G} \dot{\mathbf{q}}_{a}=\mathbf{R}_{G} \dot{\boldsymbol{\theta}}$ from (7) into (13) and (12) ones obtain

$$
\begin{gathered}
\mathbf{R}_{G} \mathbf{I}_{m} \mathbf{R}_{G} \ddot{\mathbf{q}}_{a}+\mathbf{R}_{G} \mathbf{D}_{m} \mathbf{R}_{G} \dot{\mathbf{q}}_{a}=\mathbf{R}_{G} \mathbf{K}_{m} \mathbf{i}-\boldsymbol{\tau}_{2}, \\
\mathbf{L}_{a} \frac{\mathrm{d}}{\mathrm{d} t} \mathbf{i}+\mathbf{R}_{a} \mathbf{i}=\mathbf{u}-\mathbf{K}_{e} \mathbf{R}_{G} \dot{\mathbf{q}}_{a} .
\end{gathered}
$$

Noting that, the matrix $\mathbf{B}$ has the form

$$
\mathbf{B}=\left[\begin{array}{c}
\mathbf{E}_{n \times n} \\
\mathbf{0}_{m-n, n}
\end{array}\right],
$$

so, this matrix can be multiplied to equation (14) from left. This leads to

$$
\mathbf{B} \mathbf{R}_{G} \mathbf{I}_{m} \mathbf{R}_{G} \ddot{\mathbf{q}}_{a}+\mathbf{B} \mathbf{R}_{G} \mathbf{D}_{m} \mathbf{R}_{G} \dot{\mathbf{q}}_{a}=\mathbf{B} \mathbf{R}_{G} \mathbf{K}_{m} \mathbf{i}-\mathbf{B} \tau_{2} \text {. }
$$

To eliminate the vector $\boldsymbol{\tau}_{2}$ from Eqs. (2) and (16), the matrix $\mathbf{Z}=\left[\begin{array}{ll}\mathbf{E}_{n \times n} & \mathbf{0}_{m, m-n}\end{array}\right]$ is used. Here, the following relations are satisfied

$$
\mathbf{q}_{a}=\mathbf{Z q}, \quad \dot{\mathbf{q}}_{a}=\mathbf{Z} \dot{\mathbf{q}}, \quad \ddot{\mathbf{q}}_{a}=\mathbf{Z} \ddot{\mathbf{q}} .
$$


with (17), Eqs. (15) and (16) become

$$
\begin{gathered}
\mathbf{L}_{a} \frac{\mathrm{d}}{\mathrm{d} t} \mathbf{i}+\mathbf{R}_{a} \mathbf{i}=\mathbf{u}-\mathbf{K}_{e} \mathbf{R}_{G} \mathbf{Z} \dot{\mathbf{q}} \\
\mathbf{B R}_{G} \mathbf{I}_{m} \mathbf{R}_{G} \mathbf{Z} \ddot{\mathbf{q}}+\mathbf{B} \mathbf{R}_{G} \mathbf{D}_{m} \mathbf{R}_{G} \mathbf{Z} \dot{\mathbf{q}}=\mathbf{B} \mathbf{R}_{G} \mathbf{K}_{m} \mathbf{i}-\mathbf{B} \tau_{2} .
\end{gathered}
$$

By adding two equations (2) and (19), one obtains

$$
\left(\mathbf{M}(\mathbf{q})+\mathbf{B} \mathbf{R}_{G} \mathbf{I}_{m} \mathbf{R}_{G} \mathbf{Z}\right) \ddot{\mathbf{q}}+\mathbf{C}(\mathbf{q}, \dot{\mathbf{q}}) \dot{\mathbf{q}}+\left(\mathbf{D}+\mathbf{B} \mathbf{R}_{G} \mathbf{D}_{m} \mathbf{R}_{G} \mathbf{Z}\right) \dot{\mathbf{q}}+\mathbf{g}(\mathbf{q})=\mathbf{B} \mathbf{R}_{G} \mathbf{K}_{m} \mathbf{i} .
$$

Similarly, for a closed loop system one gets

$$
\begin{aligned}
& \left(\mathbf{M}(\mathbf{q})+\mathbf{B} \mathbf{R}_{G} \mathbf{I}_{m} \mathbf{R}_{G} \mathbf{Z}\right) \ddot{\mathbf{q}}+\mathbf{C}(\mathbf{q}, \dot{\mathbf{q}}) \dot{\mathbf{q}} \\
& +\left(\mathbf{D}+\mathbf{B} \mathbf{R}_{G} \mathbf{D}_{m} \mathbf{R}_{G} \mathbf{Z}\right) \dot{\mathbf{q}}+\mathbf{g}(\mathbf{q})+\boldsymbol{\Phi}^{T}(\mathbf{q}) \boldsymbol{\lambda}=\mathbf{B} \mathbf{R}_{G} \mathbf{K}_{m} \mathbf{i}, \\
& \boldsymbol{\phi}(\mathbf{q})=\mathbf{0} \text {. }
\end{aligned}
$$

So, the dynamic model of an open loop MBS driven by electric motors is described by a set of Eqs. (20) and (18) which is in ordinary differential equations form (ODEs). And the dynamic model of a closed loop MBS driven by electric motors is described by a set of Eqs. (21) and (18) which is in DAEs form. These equations show the dynamic relationship between inputs (voltage $\mathbf{u}$ ) and outputs (motion $\mathbf{q}$ ).

\subsection{Simplified dynamic model}

Normally, the electrical time constant is much smaller than the mechanical time constant, so the approximation $\mathbf{L}_{a} d \mathbf{i} / d t \approx 0$ when $\varepsilon \geq t>0$ can be used to simplify the system of differential equations describing the system. With this approximation, solving for the current from Eq. (18) one yields

$$
\mathbf{i}=\mathbf{R}_{a}^{-1} \mathbf{u}-\mathbf{R}_{a}^{-1} \mathbf{K}_{e} \mathbf{R}_{G} \mathbf{Z} \dot{\mathbf{q}} .
$$

By substituting (22) into Eq. (20) one gets

$$
\begin{aligned}
(\mathbf{M}(\mathbf{q}) & \left.+\mathbf{B} \mathbf{R}_{G} \mathbf{I}_{m} \mathbf{R}_{G} \mathbf{Z}\right) \ddot{\mathbf{q}}+\mathbf{C}(\mathbf{q}, \dot{\mathbf{q}}) \dot{\mathbf{q}} \\
& +\left(\mathbf{D}+\mathbf{B} \mathbf{R}_{G}\left(\mathbf{D}_{m}+\mathbf{K}_{m} \mathbf{R}_{a}^{-1} \mathbf{K}_{e}\right) \mathbf{R}_{G} \mathbf{Z}\right) \dot{\mathbf{q}}+\mathbf{g}(\mathbf{q})=\mathbf{B} \mathbf{R}_{G} \mathbf{K}_{m} \mathbf{R}_{a}^{-1} \mathbf{u}
\end{aligned}
$$

Similarly, for a closed loop systems Eq. (21) becomes

$$
\begin{aligned}
& \begin{aligned}
\left(\mathbf{M}(\mathbf{q})+\mathbf{B} \mathbf{R}_{G} \mathbf{I}_{m} \mathbf{R}_{G} \mathbf{Z}\right) \ddot{\mathbf{q}}+\mathbf{C}(\mathbf{q}, \dot{\mathbf{q}}) \dot{\mathbf{q}} & +\left(\mathbf{D}+\mathbf{B} \mathbf{R}_{G}\left(\mathbf{D}_{m}+\mathbf{K}_{m} \mathbf{R}_{a}^{-1} \mathbf{K}_{e}\right) \mathbf{R}_{G} \mathbf{Z}\right) \dot{\mathbf{q}}+\mathbf{g}(\mathbf{q}) \\
& =\mathbf{B} \mathbf{R}_{G} \mathbf{K}_{m} \mathbf{R}_{a}^{-1} \mathbf{u}+\mathbf{\Phi}_{q}^{T}(\mathbf{q}) \lambda,
\end{aligned} \\
& \boldsymbol{\phi}(\mathbf{q})=\mathbf{0 .}
\end{aligned}
$$

By defining the following matrices

$$
\begin{aligned}
& \mathbf{M}_{s}(\mathbf{q})=\left(\mathbf{M}(\mathbf{q})+\mathbf{B} \mathbf{R}_{G} \mathbf{I}_{m} \mathbf{R}_{G} \mathbf{Z}\right), \quad \mathbf{C}_{s}(\mathbf{q}, \dot{\mathbf{q}})=\mathbf{C}(\mathbf{q}, \dot{\mathbf{q}}), \\
& \mathbf{D}_{s}=\left(\mathbf{D}+\mathbf{B} \mathbf{R}_{G}\left(\mathbf{D}_{m}+\mathbf{K}_{m} \mathbf{R}_{a}^{-1} \mathbf{K}_{e}\right) \mathbf{R}_{G} \mathbf{Z}\right), \quad \mathbf{g}_{s}(\mathbf{q})=\mathbf{g}(\mathbf{q}), \quad \mathbf{B}_{s}=\mathbf{B} \mathbf{R}_{G} \mathbf{K}_{m} \mathbf{R}_{a}^{-1} .
\end{aligned}
$$

Eq. (24) is rewritten in compact form as

$$
\mathbf{M}_{s}(\mathbf{q}) \ddot{\mathbf{q}}+\mathbf{C}_{s}(\mathbf{q}, \dot{\mathbf{q}}) \dot{\mathbf{q}}+\mathbf{D}_{s} \dot{\mathbf{q}}+\mathbf{g}_{s}(\mathbf{q})=\mathbf{B}_{s} \mathbf{u}+\boldsymbol{\Phi}_{q}^{T}(\mathbf{q}) \lambda
$$


Once again, the constraint equations are combined

$$
\boldsymbol{\phi}(\mathbf{q})=\mathbf{0} .
$$

Thus, the dynamic model of closed loop MBS driven by electric motors is described by a set of DAEs (26) and (27). For an open loop MBS driven by electric motors, the dynamic model is obtained by deleting the last term in Eqs. (26) and ignoring Eq. (27), because in this case the generalized coordinates $\mathbf{q}$ contains independent variable. And it is as

$$
\mathbf{M}_{s}(\mathbf{q}) \ddot{\mathbf{q}}+\mathbf{C}_{s}(\mathbf{q}, \dot{\mathbf{q}}) \dot{\mathbf{q}}+\mathbf{D}_{s} \dot{\mathbf{q}}+\mathbf{g}_{s}(\mathbf{q})=\mathbf{B}_{s} \mathbf{u} \text {. }
$$

Noting that $\mathbf{B} \mathbf{R}_{G} \mathbf{I}_{m} \mathbf{R}_{G} \mathbf{Z}$ is the symmetric constant matrix (because $\mathbf{Z}=\mathbf{B}^{T}$ ), so the Coriolis matrices $\mathbf{C}_{s}(\mathbf{q}, \dot{\mathbf{q}})$ or $\mathbf{C}(\mathbf{q}, \dot{\mathbf{q}})$ calculating from mass matrices $\mathbf{M}_{s}(\mathbf{q})$ or $\mathbf{M}(\mathbf{q})$ are the same, and skew-symmetric property of matrix $\mathbf{N}=\dot{\mathbf{M}}_{s}(\mathbf{q})-2 \mathbf{C}_{s}(\mathbf{q}, \dot{\mathbf{q}})$ is still remained.

\section{EXAMPLES}

In this section, the dynamic models of some electro-mechanical systems in engineering are presented to illustrate the proposed approach. Four systems considered here include an overhead crane (a typical underactuated system), a serial manipulator (a typical open loop MBS and full actuated system), a slider-crank mechanism and a 3RRR planar parallel robot (a typical closed loop MBS and full actuated systems).

\subsection{Overhead crane - an underactuated system}

Overhead cranes are widely used in various fields, such as heavy industries, seaports, automotive factories, and construction facilities. An overhead crane is typical underactuated system because of swing motion of the payload. In this example, the length of the cable is assumed to be constant. The system has two degrees of freedom and is driven by only one motor for the horizonal motion of the troley (Fig. 2). The parameters of mechanical system including the mass of the trolley $m_{t}$, mass of the payload $m_{p}$, and length of the cable $l$. The wheel of the trolley has radius $r_{w}$ and its mass is neglected. Let $\mathbf{q}=\left[q_{1}, q_{2}\right]^{T}$ be generalized coordinates while an active coordinate is $q_{a}=q_{1}$.

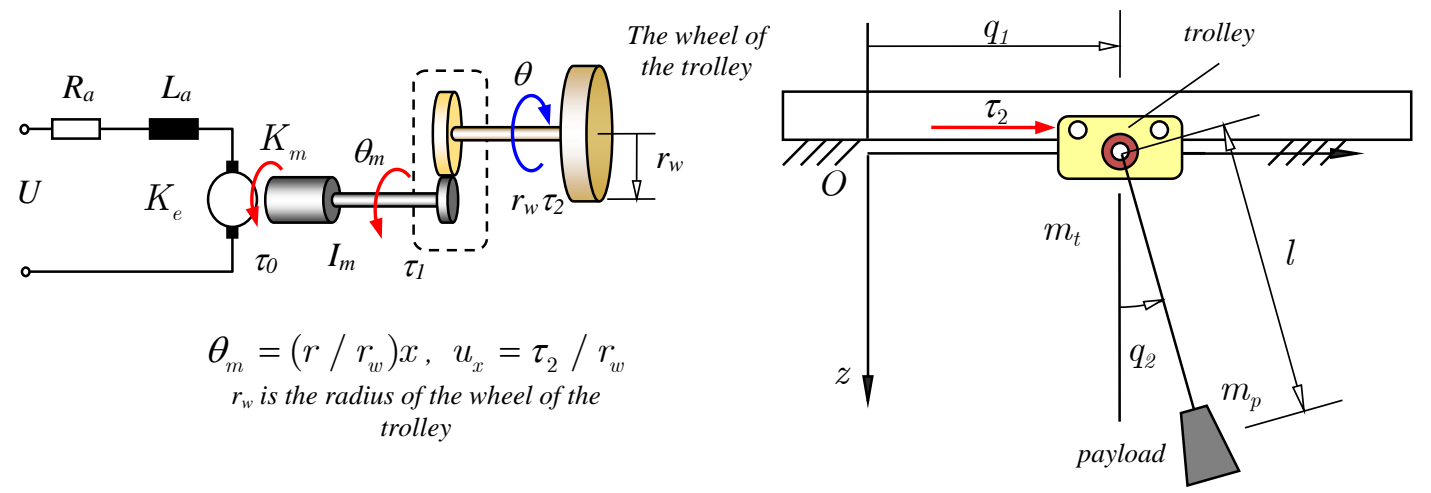

Fig. 2. The 2-dof overhead crane driven by an electric motor 
For the purpose of comparing the responses of two models: full and simplified dynamic dmodel, in this example, the differential equations of motion for these two models are given. The kinetic and potential energy of the mechanical system are given as following

$$
\begin{aligned}
& T=\frac{1}{2}\left(m_{t}+m_{p}\right) \dot{q}_{1}^{2}+m_{p} l \cos q_{2} \dot{q}_{1} \dot{q}_{2}+\frac{1}{2} m_{p} l^{2} \dot{q}_{2}^{2}, \\
& \Pi=-m_{p} g l \cos q_{2} .
\end{aligned}
$$

Full dynamic model

From the kinetic and potential energy of the system, the equation of motion in form of (3) is given with the following matrices

$$
\begin{aligned}
& \mathbf{M}(\mathbf{q})=\left[\begin{array}{cc}
m_{t}+m_{p} & m_{p} l \cos q_{2} \\
m_{p} l \cos q_{2} & m_{p} l^{2}
\end{array}\right], \quad \mathbf{C}_{s}(\mathbf{q}, \dot{\mathbf{q}})=\left[\begin{array}{cc}
0 & -m_{p} l \dot{q}_{2} \sin q_{2} \\
0 & 0
\end{array}\right], \quad \mathbf{D}=\left[\begin{array}{cc}
d_{1} & 0 \\
0 & 0
\end{array}\right], \\
& \mathbf{g}(\mathbf{q})=\left[\begin{array}{ll}
0 & m_{p} g l \sin q_{2}
\end{array}\right]^{T}, \quad \mathbf{B}=\left[\begin{array}{l}
1 \\
0
\end{array}\right], \quad \boldsymbol{\tau}_{2}=\boldsymbol{\tau}_{2} .
\end{aligned}
$$

Eqs. (7), (11) and (12) for this example are written as follows

$$
\begin{array}{lll}
r \dot{q}_{1} / r_{w}=\dot{\theta}_{m}, \quad \tau_{2}=\left(r / r_{w}\right) \tau_{1} & \Rightarrow \quad \mathbf{R}_{G}=\left[r / r_{w}\right] \\
I_{m} \ddot{\theta}_{m}+d_{m} \dot{\theta}_{m}=K_{m} i-\tau_{1} \quad \text { or } & I_{m}\left(r / r_{w}\right)^{2} \ddot{q}_{1}+d_{m}\left(r / r_{w}\right)^{2} \dot{q}_{1}=\left(r / r_{w}\right) K_{m} i-\tau_{2}, \\
L_{a} \frac{\mathrm{d}}{\mathrm{d} t} i+R_{a} i=u-K_{e} \dot{\theta}_{m} \quad \text { or } & L_{a} \frac{\mathrm{d}}{\mathrm{d} t} i+R_{a} i=u-K_{e}\left(r / r_{w}\right) \dot{q}_{1} .
\end{array}
$$

In this case, $\mathbf{Z}=\left[\begin{array}{ll}1 & 0\end{array}\right]$. The combination of Eqs. (18) and (20) is rewritten in matrix form as follows

$$
\mathbf{A}(\mathbf{q}) \ddot{\mathbf{q}}+\mathbf{h}(\mathbf{q}, \dot{\mathbf{q}})=\mathbf{B u}, \quad \mathbf{u}=U,
$$

with $\dot{q}_{3}=i$ and $\ddot{q}_{3}=\dot{i}$ and

$$
\begin{gathered}
\mathbf{q}=\left[\begin{array}{l}
q_{1} \\
q_{2} \\
q_{3}
\end{array}\right], \quad \mathbf{A}(\mathbf{q})=\left[\begin{array}{ccc}
m_{t}+m_{p}+\left(r / r_{w}\right)^{2} I_{m} & m_{p} l \cos q_{2} & 0 \\
m_{p} l \cos q_{2} & m_{p} l^{2} & 0 \\
0 & 0 & L_{a}
\end{array}\right], \\
\mathbf{h}(\mathbf{q}, \dot{\mathbf{q}})=\left[\begin{array}{c}
-m_{p} l \dot{q}_{2}^{2} \sin q_{2}+\left[d_{1}+d_{m}\left(r / r_{w}\right)^{2}\right] \dot{q}_{1}-\left(r / r_{w}\right) K_{m} \dot{q}_{3} \\
m_{p} g l \sin q_{2} \\
K_{e}\left(r / r_{w}\right) \dot{q}_{1}+R_{a} \dot{q}_{3}
\end{array}\right], \quad \mathbf{B}=\left[\begin{array}{l}
0 \\
0 \\
1
\end{array}\right] .
\end{gathered}
$$

Simplified dynamic model

By using the approximation $L_{a} \dot{i}=L_{a} \ddot{q}_{3} \approx 0$, the simplified model for the crane in the form of (28) is given as

$$
\mathbf{M}_{s}(\mathbf{q}) \ddot{\mathbf{q}}+\mathbf{C}_{s}(\mathbf{q}, \dot{\mathbf{q}}) \dot{\mathbf{q}}+\mathbf{D}_{s} \dot{\mathbf{q}}+\mathbf{g}_{s}(\mathbf{q})=\mathbf{B}_{s} \mathbf{u},
$$


with the following matrices

$$
\begin{aligned}
& \mathbf{M}_{s}(\mathbf{q})=\left[\begin{array}{cc}
m_{t}+m_{p}+r^{2} I_{m} / r_{w}^{2} & m_{p} l \cos q_{2} \\
m_{p} l \cos q_{2} & m_{p} l^{2}
\end{array}\right], \quad \mathbf{C}_{s}(\mathbf{q}, \dot{\mathbf{q}})=\left[\begin{array}{cc}
0 & -m_{p} l \dot{q}_{2} \sin q_{2} \\
0 & 0
\end{array}\right], \\
& \mathbf{g}(\mathbf{q})=\left[\begin{array}{ll}
0 & m_{p} g l \sin q_{2}
\end{array}\right]^{T}, \\
& \mathbf{D}_{s}=\left[\begin{array}{cc}
d_{1}+d_{m}\left(r / r_{w}\right)^{2}+r^{2} K_{m} R_{a}^{-1} K_{e} r_{w}^{-2} & 0 \\
0 & 0
\end{array}\right], \quad \mathbf{B}_{s}=\left[\begin{array}{c}
r K_{m} R_{a}^{-1} r_{w}^{-1} \\
0
\end{array}\right], \quad \mathbf{u}=U .
\end{aligned}
$$

\subsection{Equations of motion of a serial manipulator: 2-dof open loop MBS}

In this example, we consider a 2-dof planar manipulator moving in a vertical plane (Fig. 3). The manipulator is driven by two electric motors, motor 1 is fixed on the ground and motor 2 is placed on link 1 . The motions of the motor shaft are transmitted to the link by the gear-box transmission. The parameters of two links including masses, length, distance from joint to center of mass and moment of inertia about its center are $m_{1}, m_{2}$, $l_{1}, l_{2}, a_{1}, a_{2}, I_{C 1}, I_{C 2}$, respectively.

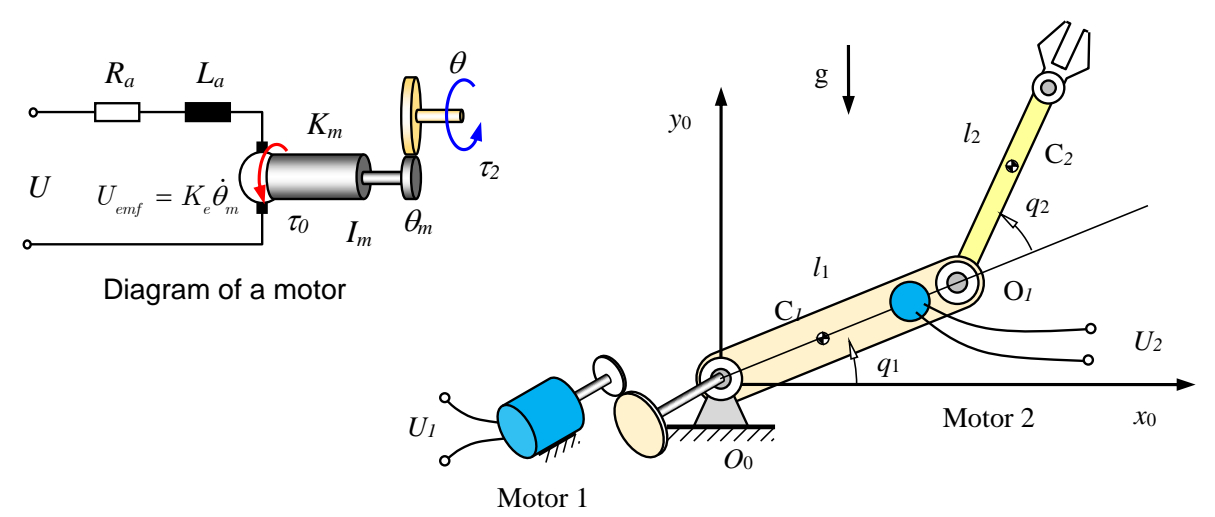

Fig. 3. The 2-dof manipulator driven by electric motors

The system has two degrees of freedom and the the generalized coordinates are defined as

$$
\mathbf{q}=\left[q_{1}, q_{2}\right]^{T}, \text { so } m=n=2
$$

The kinetic and potential energy of the system are given as following

$$
\begin{aligned}
T= & 0.5\left[I_{C 1}+m_{1} a_{1}^{2}+I_{C 2}+m_{2}\left(l_{1}^{2}+a_{2}^{2}+2 l_{1} a_{2} \cos q_{2}\right)\right] \dot{q}_{1}^{2} \\
& +\left[I_{C 2}+m_{2}\left(a_{2}^{2}+l_{1} a_{2} \cos q_{2}\right)\right] \dot{q}_{1} \dot{q}_{2}+0.5\left(I_{C 2}+m_{2} a_{2}^{2}\right) \dot{q}_{2}^{2} . \\
\Pi= & m_{1} g a_{1} \sin q_{1}+m_{2} g\left(l_{1} \sin q_{1}+a_{2} \sin \left(q_{1}+q_{2}\right)\right) .
\end{aligned}
$$

Matrices $\mathbf{B}$ and $\mathbf{Z}$ are $\mathbf{B}=\operatorname{diag}([1,1]), \mathbf{Z}=\operatorname{diag}([1,1])$.

From the kinetic, potential energy of the mechanism, the parameters of the electric motor, gear transmission ratio, the equation of motion in form of (23) is given with the 
following matrices

$$
\begin{aligned}
& \mathbf{M}_{s}(\mathbf{q})=\left[\begin{array}{cc}
I_{C 1}+m_{1} a_{1}^{2}+I_{C 2}+m_{2}\left(l_{1}^{2}+a_{2}^{2}+2 l_{1} a_{2} \cos q_{2}\right)+r^{2} I_{m} & I_{C 2}+m_{2}\left(a_{2}^{2}+l_{1} a_{2} \cos q_{2}\right) \\
I_{C 2}+m_{2}\left(a_{2}^{2}+l_{1} a_{2} \cos q_{2}\right) & I_{C 2}+m_{2} a_{2}^{2}+r^{2} I_{m}
\end{array}\right], \\
& \mathbf{C}_{s}(\mathbf{q}, \dot{\mathbf{q}})=\left[\begin{array}{cc}
-m_{2} l_{1} a_{2} \dot{q}_{2} \sin q_{2} & -m_{2} l_{1} a_{2}\left(\dot{q}_{1}+\dot{q}_{2}\right) \sin q_{2} \\
m_{2} l_{1} a_{2} \dot{q}_{1} \sin q_{2} & 0
\end{array}\right], \\
& \mathbf{D}_{s}=\left[\begin{array}{cc}
d_{1}+r^{2} K_{m} R_{a}^{-1} K_{e} & 0 \\
0 & d_{2}+r^{2} K_{m} R_{a}^{-1} K_{e}
\end{array}\right], \\
& \mathbf{g}_{s}=\left[\begin{array}{cc}
m_{1} g a_{1} \cos q_{1}+m_{2} g\left[l_{1} \cos q_{1}+a_{2} \cos \left(q_{1}+q_{2}\right)\right] \\
m_{2} g a_{2} \cos \left(q_{1}+q_{2}\right)
\end{array}\right], \\
& \mathbf{B}_{s}=\left[\begin{array}{cc}
r K_{m} R_{a}^{-1} & 0 \\
0 & r K_{m} R_{a}^{-1}
\end{array}\right], \\
& \mathbf{u}=\left[\begin{array}{c}
U_{1} \\
U_{2}
\end{array}\right]
\end{aligned}
$$

\subsection{Equations of motion of the slider-crank mechanism: 1 dof closed loop MBS}

Lets consider a slider-crank mechanism moving in the vertical plane, the crank is driven by an electric motor through gear-box (Fig. 4). This is a closed loop MBS driven by an electric motor. The mechanism consists of a crank $O A$ with mass $m_{1}$, moment of inertia $I_{C 1}$, length $l_{1}$, and $O C_{1}=a_{1}$; a connecting rod $A B$ with mass $m_{2}$, moment of inertia $I_{C 2}$, length $l_{2}$, and $A C_{2}=a_{2}$; and a slider $\mathrm{B}$ with mass $m_{3}$. The parameters of electric motor include $I_{m}\left(\mathrm{~kg} \cdot \mathrm{m}^{2}\right), K_{m}(\mathrm{Nm} / \mathrm{A}), K_{e}(\mathrm{Vs} / \mathrm{rad}), R_{a}(\mathrm{Ohm})$ and transmission ratio of gear-box is $r$.

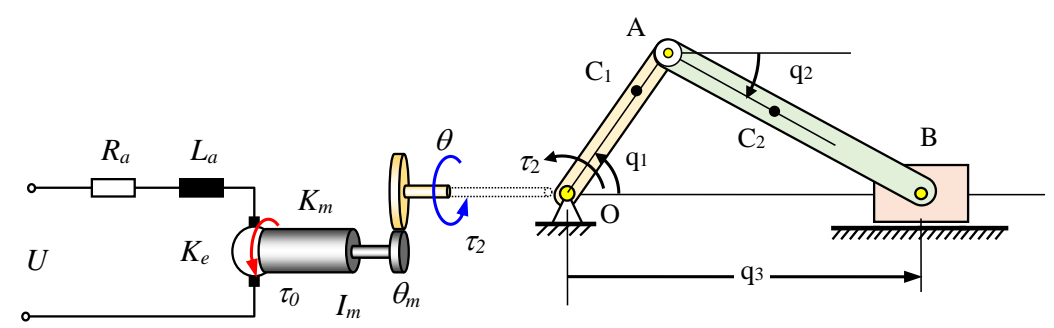

Fig. 4. The slider-crank mechanism driven by an electric motor

The system has only one degree of freedom and the generalized coordinates are defined as

$$
\mathbf{q}=\left[q_{1}, q_{2}, q_{3}\right]^{T}, \text { so } m=3, n=1
$$

Matrices $\mathbf{B}$ and $\mathbf{Z}$ are $\mathbf{B}=[1,0,0]^{T}, \mathbf{Z}=[1,0,0]$.

The kinetic and potential energy of the system are given as following:

$$
\begin{aligned}
& T=\frac{1}{2}\left(I_{C 1}+m_{1} a_{1}^{2}+m_{2} l_{1}^{2}\right) \dot{q}_{1}^{2}-m_{2} l_{1} a_{2} \cos \left(q_{1}+q_{2}\right) \dot{q}_{1} \dot{q}_{2}+\frac{1}{2}\left(I_{C 2}+m_{2} a_{2}^{2}\right) \dot{q}_{2}^{2}+\frac{1}{2} m_{3} \dot{q}_{3}^{2}, \\
& \Pi=m_{1} g a_{1} \sin q_{1}+m_{2} g\left(l_{1} \sin q_{1}-a_{2} \sin q_{2}\right) .
\end{aligned}
$$


From the kinetic and potential energy of the mechanism, the parameters of the electric motor, gear transmission ratio, the equation of motion in form of (26) is given with the following matrices

$$
\begin{aligned}
& \mathbf{M}_{\mathbf{s}}(\mathbf{q})=\left[\begin{array}{ccc}
I_{C 1}+m_{1} a_{1}^{2}+m_{2} l_{1}^{2}+r^{2} I_{m} & -m_{2} l_{1} a_{2} \cos \left(q_{1}+q_{2}\right) & 0 \\
-m_{2} l_{1} a_{2} \cos \left(q_{1}+q_{2}\right) & I_{C 2}+m_{2} a_{2}^{2} & 0 \\
0 & 0 & m_{3}
\end{array}\right], \\
& \mathbf{C}_{\mathbf{s}}(\mathbf{q}, \dot{\mathbf{q}})=\left[\begin{array}{ccc}
0 & m_{2} l_{1} a_{2} \dot{q}_{2} \sin \left(q_{1}+q_{2}\right) & 0 \\
m_{2} l_{1} a_{2} \dot{q}_{1} \sin \left(q_{1}+q_{2}\right) & 0 & 0 \\
0 & 0 & 0
\end{array}\right], \\
& \mathbf{D}_{s}=\operatorname{diag}\left(\left[d+r^{2} K_{m} R_{a}^{-1} K_{e}, 0,0\right]\right), \\
& \mathbf{g}_{\mathbf{s}}(\mathbf{q})=\left[g\left(m_{1} a_{1} \cos q_{1}+m_{2} l_{1} \cos q_{1}\right),-m_{2} g a_{2} \cos q_{2}, 0\right]^{T}, \\
& \mathbf{B}_{s}=\left[r K_{m} R_{a}^{-1}, 0,0\right]^{T}, \quad \mathbf{u}=U .
\end{aligned}
$$

The constraint equations are given in the below form

$$
\begin{aligned}
& \phi_{1}(\mathbf{q})=l_{1} \cos q_{1}+l_{2} \cos q_{2}-q_{3}=0, \\
& \phi_{2}(\mathbf{q})=l_{1} \sin q_{1}-l_{2} \sin q_{2}=0,
\end{aligned}
$$

and the Jacobian matrix is

$$
\boldsymbol{\Phi}_{q}(\mathbf{q})=\left[\begin{array}{ccc}
-l_{1} \sin q_{1} & -l_{2} \sin q_{2} & -1 \\
l_{1} \cos q_{1} & -l_{2} \cos q_{2} & 0
\end{array}\right]
$$

\subsection{The 3RRR planar parallel robot: 3 dof closed loop MBS, full-actuated system}

Fig. 5 shows the considered 3RRR planar parallel robot moving in a horizontal plane. The fixed base and the moving platform are the two equilateral triangles $\mathrm{O}_{1} \mathrm{O}_{2} \mathrm{O}_{3}$ and $B_{1} B_{2} B_{3} ; L_{0}$ and $L_{1}$ are the lengths of two triangles, respectively. It also has three legs and each leg has the same two links $O_{i} A_{i}=l_{1}, A_{i} B_{i}=l_{2}$. Three active joints are actuated by three electric motors through gear-box transmission. The system has three degrees of freedom and the redundant generalized coordinates are defined as $\mathbf{q}=\left[\theta^{T}, \mathbf{x}^{T}\right]^{T}=$ $\left[\theta_{1}, \theta_{2}, \theta_{3}, x_{C}, y_{C}, \varphi\right]^{T}$, so $n=3$, and $m=6$.

To make the dynamic model simple, masses $m_{2}$ of the connecting links $A_{i} B_{i}$ are considered as concentrated at the end of links. The kinetic and potential energy of the mechanical system are given as following

$$
\begin{aligned}
& T=\frac{1}{2} \sum_{k=1}^{3}\left(I_{C 1}+\frac{1}{4} m_{1} l_{1}^{2}+\frac{1}{2} m_{2} l_{1}^{2}\right) \dot{\theta}_{k}^{2}+\frac{1}{2}\left(m_{3}+3 \cdot \frac{1}{2} m_{2}\right)\left(\dot{x}_{C}^{2}+\dot{y}_{C}^{2}\right)+\frac{1}{2}\left(I_{C 3}+3 \cdot \frac{1}{2} m_{2} b^{2}\right) \dot{\varphi}^{2}, \\
& \Pi=0 .
\end{aligned}
$$

Matrices $\mathbf{B}$ and $\mathbf{Z}$ are

$$
\mathbf{B}=\left[\begin{array}{l}
\mathbf{E}_{3 \times 3} \\
\mathbf{0}_{3 \times 3}
\end{array}\right], \quad \mathbf{Z}=\left[\begin{array}{ll}
\mathbf{E}_{3 \times 3} & \mathbf{0}_{3 \times 3}
\end{array}\right] .
$$




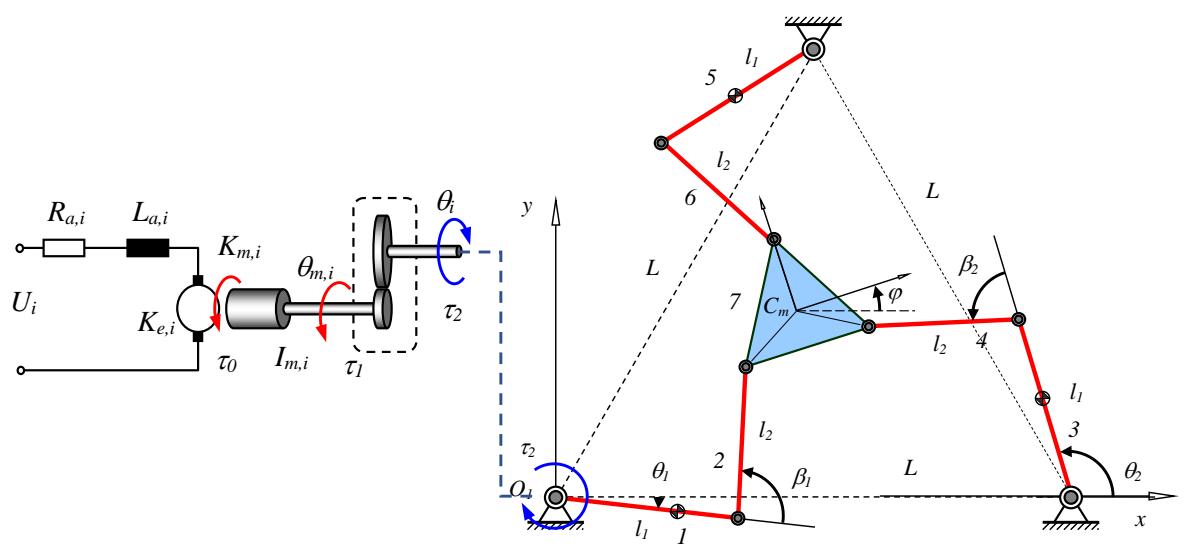

Fig. 5. Model of an electric motor with gearbox and a 3RRR planar parallel robot

From kinetic, potential energy of the mechanism, the parameters of the electric motor, gear transmission ratio, the equation of motion in form of (26) is given with the following matrices

$$
\begin{aligned}
& \mathbf{M}_{s}=\operatorname{diag}\left(\left[\left(I_{m} r^{2}+I_{C 1}+\frac{1}{4} m_{1} l_{1}^{2}+\frac{1}{2} m_{2} l_{1}^{2}\right)[1,1,1],\left(m_{3}+3 \cdot \frac{1}{2} m_{2}\right)[1,1], I_{C 3}+3 \cdot \frac{1}{2} m_{2} b^{2}\right]\right), \\
& \mathbf{C}_{s}(\mathbf{q}, \dot{\mathbf{q}})=\mathbf{0}_{6 \times 6}, \quad \mathbf{D}_{s}=\operatorname{diag}\left([1,1,1,0,0,0] r^{2} K_{m} R_{a}^{-1} K_{e}\right), \quad \mathbf{g}_{s}(\mathbf{q})=\mathbf{0}_{6 \times 1}, \\
& \mathbf{B}_{s}=\left[\begin{array}{c}
r K_{m} R_{a}^{-1} \mathbf{E}_{3 \times 3} \\
\mathbf{0}_{3 \times 3}
\end{array}\right], \quad \mathbf{u}=\left[U_{1}, U_{2}, U_{3}\right]^{T}, \quad \boldsymbol{\Phi}_{q}^{T}(\mathbf{q})=\left(\frac{\partial \mathbf{f}}{\partial \mathbf{q}}\right)^{T}, \quad \lambda=\left[\lambda_{1}, \lambda_{2}, \lambda_{3}\right]^{T} .
\end{aligned}
$$

The constraint equations are given from distanced conditions between two points $A_{i}$ and $B_{i}$

$$
f_{i}=\left(\mathbf{r}_{B_{i}}-\mathbf{r}_{A_{i}}\right)^{T}\left(\mathbf{r}_{B_{i}}-\mathbf{r}_{A_{i}}\right)-l_{2}^{2}=0, \quad i=1,2,3
$$

where $\mathbf{r}_{A_{i}}=\left[\begin{array}{c}x_{O_{i}}+l_{1} \cos \theta_{i} \\ y_{O_{i}}+l_{1} \sin \theta_{i}\end{array}\right], \mathbf{r}_{B_{i}}=\left[\begin{array}{l}x_{C}+b \cos \left(\varphi+\alpha_{i}\right) \\ y_{C}+b \sin \left(\varphi+\alpha_{i}\right)\end{array}\right]$, with $\alpha_{1,2,3}=\left[\frac{7}{6} \pi,-\frac{1}{6} \pi, \frac{1}{2} \pi\right]$. Rewrite the constraint equations (40) as follows

$$
\mathbf{f}(\mathbf{q})=\mathbf{f}(\boldsymbol{\theta}, \mathbf{x})=\mathbf{0}, \quad \mathbf{f} \in \mathbb{R}^{3},
$$

and the Jacobian matrix is

$$
\boldsymbol{\Phi}_{q}(\mathbf{q})=\frac{\partial \mathbf{f}}{\partial \mathbf{q}}
$$

\section{VALIDATION OF SIMPLIFIED DYNAMIC MODEL BY NUMERICAL SIMULATIONS}

To validate the correctness and acceptability of the simplified motion equations of the electromechanical system, numerical simulations for the models of an overhead crane and a slider-crank mechanism are carried out. Two numerical simulations for each system are implemented in Matlab: one with the full dynamic model and the other with the simplified one. 


\subsection{Numerical simulations for a crane model}

The dynamic responses of an overhead crane under applying of a voltage $U$ on the motor is investigated in this subsection. The response are obtained by solving the ordinary differential equations (33) and (34), respectively. The simulations are carried out with the following parameters:

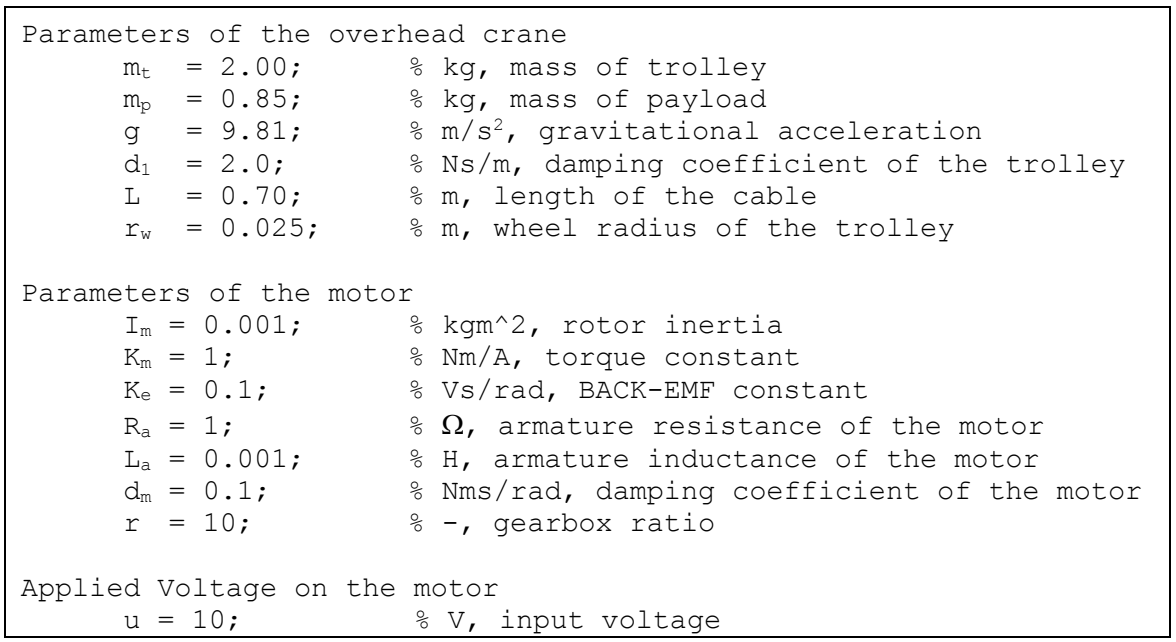

The simulation results are shown in Figs. 6-8 including motor current $i(t)$, velocity of the trolley $v(t)$, and swing-angle of the cable $q_{2}(t)$.

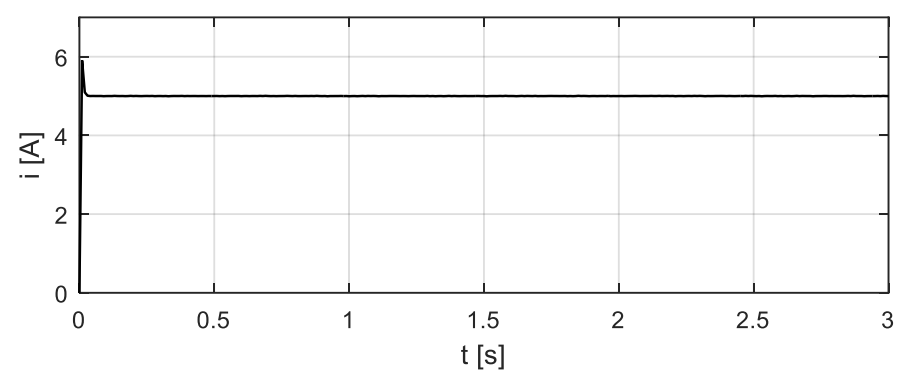

Fig. 6. Time history of current in the motor (full model)

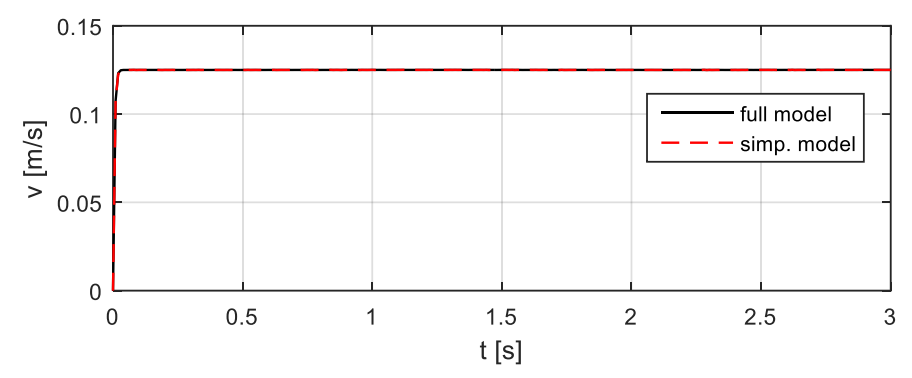

Fig. 7. Time history of velocity of the trolley: solid-black lines (full model) and dashed lines (simplified model) 


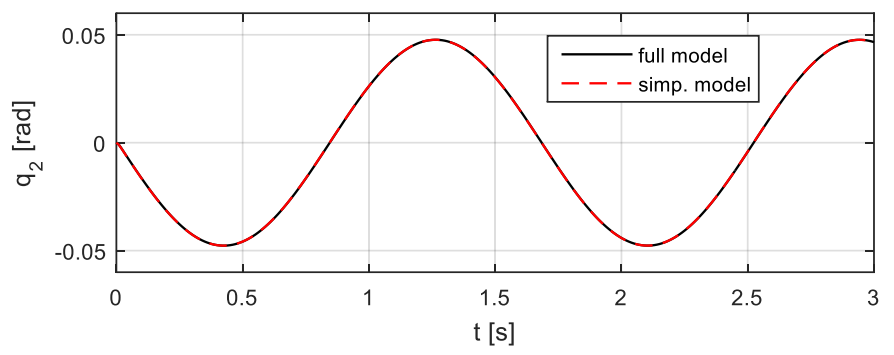

Fig. 8. Time history of swing angle: solid-black lines (full model) and dashed lines (simplified model)

The simulation results show that the electric current changes only in a very short initial time, then it keeps a constant value (Fig. 6). Time responses of trolley and swingangle of the cable in two cases (full and simplified model) are almost identical (Figs. 7 and 8 ).

\subsection{Numerical simulations for a slider-crank mechanism}

A slider-crank mechanism driven by an electric motor (Fig. 4) is chosen for numerical experiment in this subsection. The length of the crank is smaller than the length of the connecting rod, $L_{1}<L_{2}$. With this choice there is no singular point in the forward dynamic simulation of the mechanism. In addition, the dynamic simulation of this mechanism requires solving a DAEs that is accompanied by the constraint stabilization. In the dynamic simulation, the method of the Lagrangian multiplier elimination and Baumgartes stabilization technique are exploited [29].

Two models of the mechanism, the full and simplified one, are simulated. These are corresponding to the solving of the DAEs (18) and (21), and DAEs (26) and (27), respectively. The simulations are carried out with the following parameters:

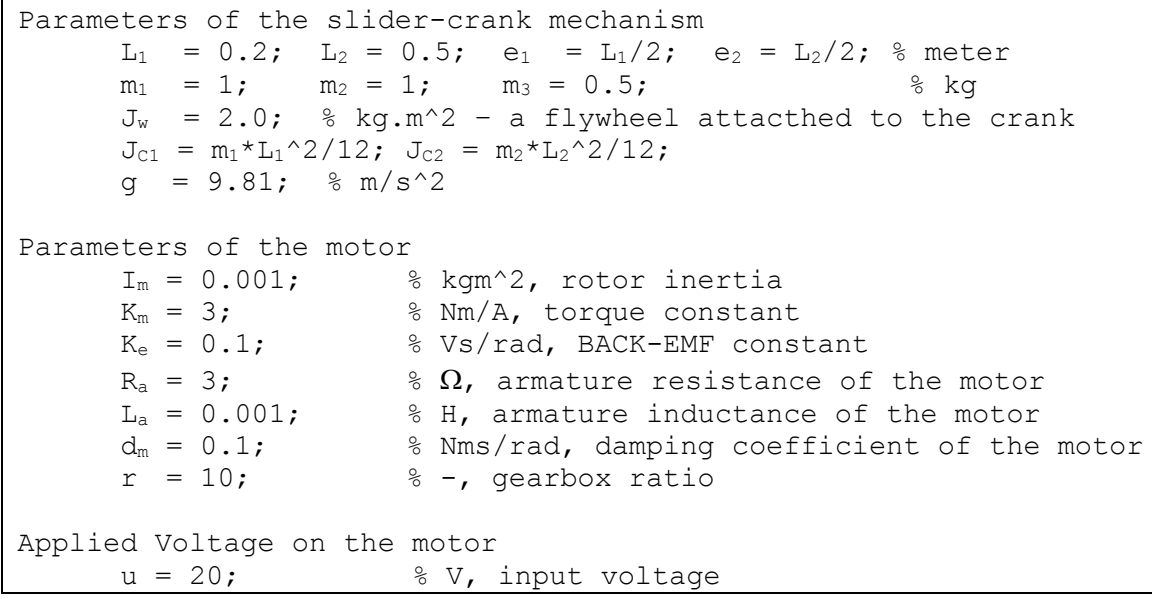


The simulation results are shown in Figs. 9-12 including motor current $i(t)$, angular velocity of the crank $\dot{q}_{1}(t)$, the angular position of the connecting rod $q_{2}(t)$ and the position of the slider, $q_{3}(t)$.

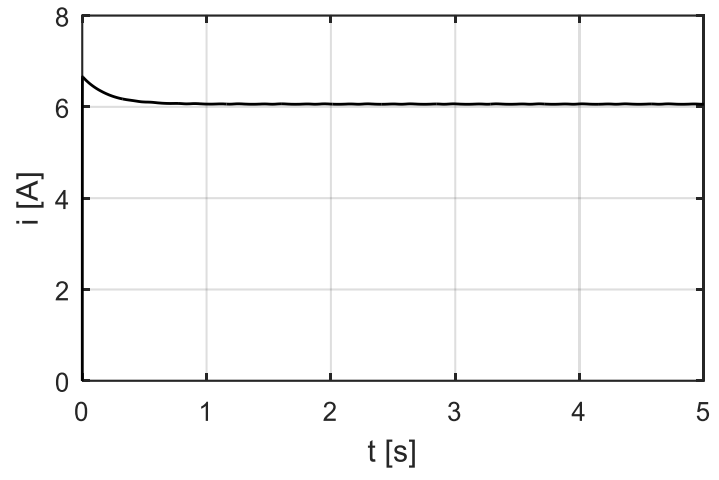

Fig. 9. Time history of current in the motor (full model)

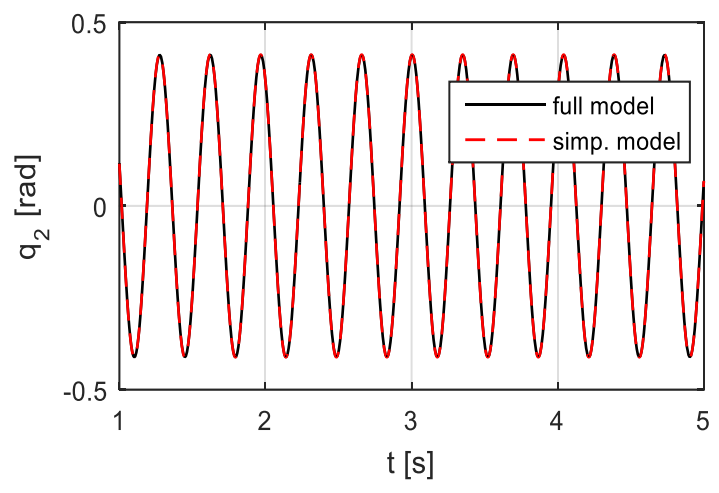

Fig. 11. Time history of the angular position of the connecting rod

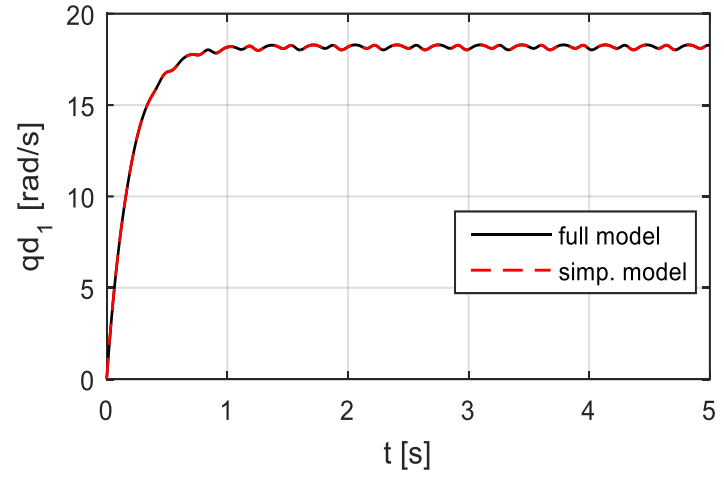

Fig. 10. Time history of the angular velocity of the crank

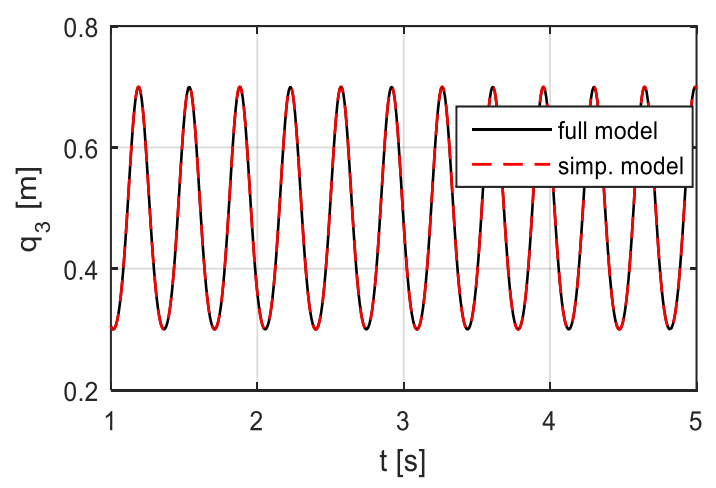

Fig. 12. Time history of the position of the slider

The simulation results show that the electric current changes only in the first half second, then it keeps the constant value (Fig. 9). The motions of the mechanism in two cases (full and simplified model) are almost identical (Fig. 10-12).

By numerical investigation with two systems: the overhead crane and the slidercrank mechanism, it can be concluded that neglecting the change of current is acceptable. Therefore, instead of using the full dynamic model, we only need to use the simplified dynamic model with a smaller number of equations than the number of equations in case of the full description. 


\section{CONCLUSION}

This paper presents a unified and efficient modelling methodology for establishing of motion equations of electromechanical systems in engineering. The whole system is split into mechanical sub-system, normally a rigid multibody system with an open or/and a closed loop, and electric motors. These two subsystems are coupled by massless gear-box transmission. The dynamic equations of each subsystem are written in matrix form. Therefore, it is easy to get the final equations by matrix multiplications and additions. The efficiency of the proposed approach is illustrated by the construction of equations for four electromechanical systems in engineering including under-actuated, full-actuated, open loop as well as closed loop systems. The obtained model in this study included electrical and mechanical parts, so it described better the real electromechanical system than in the case of the mechanical system only. In this way, it is easy to apply to other mechanical systems driven by electric motors. In addition, the simplified dynamic model obtained by neglecting variation in current of motors is validated by numerical investigation with an overhead crane and a slider-crank mechanism. The dynamic models obtained by the approach in this paper are easily extended for mechatronic systems by adding feedback controllers.

\section{REFERENCES}

[1] A. A. Shabana. Dynamics of multibody systems. Cambridge University Press, 3rd edition, (2005).

[2] T. R. Kane and D. A. Levinson. Dynamics: Theory and applications. McGraw-Hill, New York, (1985).

[3] J. Wittenburg. Dynamics of multibody systems. Springer, (2007).

[4] F. Amirouche. Fundamentals of multibody dynamics - Theory and applications. Birkhaeuser Boston, (2006).

[5] R. M. Murray, Z. Li, and S. S. Sastry. A mathematical introduction to robotic manipulation. CRC Press, (2017).

[6] R. Von Schwerin. Multibody system simulation: Numerical methods, algorithms, and software. Springer-Verlag Berlin Heidelberg, (1999).

[7] F. C. Moon. Applied dynamics with applications to multibody and mechatronic systems. John Wiley \& Sons, (1998).

[8] R. N. Jazar. Advanced dynamics: Rigid body, multibody, and aerospace applications. John Wiley \& Sons, (2011).

[9] N. V. Khang. Dynamics of multibody systems. Science and Technology Publishing House, Hanoi, (2007). (in Vietnamese).

[10] L.-W. Tsai. Robot analysis: the mechanics of serial and parallel manipulators. John Wiley \& Sons, (1999).

[11] J. Angeles and J. Angeles. Fundamentals of robotic mechanical systems, Vol. 2. Springer, (2002).

[12] J. G. De Jalon and E. Bayo. Kinematic and dynamic simulation of multibody systems: the real-time challenge. Springer-Verlag New York Inc, (1994).

[13] M. Ceccarelli. Fundamentals of mechanics of robotic manipulation, Vol. 27. Springer Science \& Business Media, (2004).

[14] L. Sciavicco and B. Siciliano. Modelling and control of robot manipulators. Springer Science \& Business Media, (2012). 
[15] M. W. Spong, S. Hutchinson, and M. Vidyasagar. Robot modeling and control, Vol. 3. Wiley, New York, (2006).

[16] J.-P. Merlet. Parallel robots, Vol. 208. Springer Science \& Business Media, (2006).

[17] N. V. Khang. Kronecker product and a new matrix form of Lagrangian equations with multipliers for constrained multibody systems. Mechanics Research Communications, 38, (4), (2011), pp. 294-299. https://doi.org/10.1016/j.mechrescom.2011.04.004.

[18] N. V. Khang and D. T. Tung. A contribution to the dynamic simulation of robot manipulator with the software RobotDyn. Vietnam Journal of Mechanics, 26, (4), (2004), pp. 215-225. https://doi.org/10.15625/0866-7136/26/4/5705.

[19] N. V. Khang. Partial derivative of matrix functions with respect to a vector variable. Vietnam Journal of Mechanics, 30, (4), (2008), pp. 269-279. https://doi.org/10.15625/0866$7136 / 30 / 4 / 5632$.

[20] C. A. My and V. M. Hoan. Kinematic and dynamic analysis of a serial manipulator with local closed loop mechanisms. Vietnam Journal of Mechanics, 41, (2), (2019), pp. 141-155. https://doi.org/10.15625/0866-7136/13073.

[21] L. Sass, J. McPhee, C. Schmitke, P. Fisette, and D. Grenier. A comparison of different methods for modelling electromechanical multibody systems. Multibody System Dynamics, 12, (3), (2004), pp. 209-250. https://doi.org/10.1023/B:MUBO.0000049196.78726.da.

[22] M. Scherrer and J. McPhee. Dynamic modelling of electromechanical multibody systems. Multibody System Dynamics, 9, (1), (2003), pp. 87-115. https://doi.org/10.1023/A:1021675422011.

[23] M. Kim, W. Moon, D. Bae, and I. Park. Dynamic simulations of electromechanical robotic systems driven by DC motors. Robotica, 22, (5), (2004), pp. 523-531. https://doi.org/10.1017/S0263574704000177.

[24] J. McPhee, C. Schmitke, and S. Redmond. Dynamic modelling of mechatronic multibody systems with symbolic computing and linear graph theory. Mathematical and Computer Modelling, 10, (1), (2004), pp. 1-23. https://doi.org/10.1080/13873950412331318044.

[25] J. Moreno-Valenzuela, R. Campa, and V. Santibáñez. Model-based control of a class of voltage-driven robot manipulators with non-passive dynamics. Computers E Electrical Engineering, 39, (7), (2013), pp. 2086-2099. https://doi.org/10.1016/j.compeleceng.2013.06.006.

[26] M. H. Hiller and K. Hirsch. Multibody system dynamics and mechatronics. Journal of Applied Mathematics and Mechanics/Zeitschrift für Angewandte Mathematik und Mechanik, 86, (2), (2006), pp. 87-109. https://doi.org/10.1002/zamm.200510253.

[27] F. L. Lewis, D. M. Dawson, and C. T. Abdallah. Robot manipulator control: Theory and practice. CRC Press, (2003).

[28] Y. Yu and Z. Mi. Dynamic modeling and control of electromechanical coupling for mechanical elastic energy storage system. Journal of Applied Mathematics, 2013, (2013). https://doi.org/10.1155/2013/603063.

[29] J. Baumgarte. Stabilization of constraints and integrals of motion in dynamical systems. Computer Methods in Applied Mechanics and Engineering, 1, (1), (1972), pp. 1-16. https://doi.org/10.1016/0045-7825(72)90018-7. 